ИЗВЕСТИЯ АКАДЕМИИ НАУК ЭСТОНСКОИ ССР. ТОМ ХІV СЕРИЯ ФИЗИКО-МАТЕМАТИЧЕСКИХ И ТЕХНИЧЕСКИХ НАУК. 1965, № 1

\title{
B. ХНЖНЯКОВ
}

\section{ТЕОРИЯ КВАЗИЛИНЕИЧАТЫХ ЭЛЕКТРОННОКОЛЕБАТЕЛЬНЫХ СПЕКТРОВ И ЭФФЕКТА МЕССБАУЭРА С УЧЕТОМ АНГАРМОНИЗМА КОЛЕБАНИИ}

В последнее время в связи с разработкой кристаллических квантовых генераторов света стали актуальными вопросы детальной колебательной структуры электронноколебательных спектров примесных центров кристаллов. Открытие эффекта Мессбауэра также повысило интерес к проблеме многофононных переходов. Аналогия задач нахождения спектра $\gamma$-квантов в эффекте Мессбауэра и электронноколебательных спектров примесных центров кристаллов позволила объединить теории обоих названных явлений $[1-3]$.

В приближениях *, обычно используемых в теориях обоих явлений, ширина бесфононных линий (в ЭМ это линия Мессбауэра, в электронноколебательных спектрах это чисто-электронная линия) равна радиационной ширине возбужденного уровня, что составляет $10^{-7}-10^{-11}$ эв либо меньше. Также радиационную ширину. в этих приближениях имеют колебательные пювторения бесфононных линий, вызванные изменением состояния локальных колебаний. На столь узкие линии могут существенно влиять многие даже весьма слабые взаимодействия в кристалле. Поэтому должны быть рассмотрены более общие модели.

В данной работе ** рассматривается влияние ангармонизма колебаний на эффект Мессбауэра и электронноколебательные спектры. Учитываются как кристаллические, так и локальные колебания. Расчеты проводятся во втором приближении по оператору ангармонизма третьего порядка и в первом приближении по оператору ангармонизма четвертого порядка. Основное внимание обращено на положение, ширину и структуру квазилиний; ангармонические поправки к вероятностям переходов не рассматриваются (учет влияния ангармонизма на вероятность линии Мессбауэра при отсутствии локальных колебаний см. в [5]).

\section{1. Общие формулы}

Остановимся прежде всего на электронноколебательных переходах в примесном центре.

* Такими приближениями являются: адиабатическое, гармоническое по колебаниям решетки, независимость электронного либо ядерного матричного элемента от координат колебаний кристалла. Кроме того, в эффекте Мессбауэра пренебрегается изменением адиабатического потенциала при $\boldsymbol{\gamma}$-переходе, а в электронноколебательных переходах - импульсом отдачи фотона и квадратичными членами в разности адиабатических потенциалов.

** Результаты работы частично перекрываются с данными, приведенными в статье М. А. Кривоглаза [4]. 
Вероятность излучения $(\tau(\omega))$ и коэффициент поглощения $(\chi(\omega))$ света с частотой $\omega$ примесным центром определяются следующими формулами [6]:

$$
\begin{aligned}
& \tau(\omega)=\frac{4 n(\omega) \omega^{4}}{3 c^{2}} I_{y}(\omega), \\
& x(\omega)=\frac{4 \pi^{2} \omega}{3 \hbar c n(\omega)} I_{\chi}(\omega),
\end{aligned}
$$

где $n(\omega)$ - показатель преломления света частоты $\omega$ в кристалле; функции $I_{y}(\omega)$ и $I_{x}(\omega)$, учитывающие детальное квантовомеханическое распределение вероятностей электронноколебательных переходов, определяются единой формулой $I(\omega)$ :

$$
I(\omega)=\frac{1}{2 \pi} \int_{-\infty}^{\infty} e^{-i \omega t-\gamma_{0}|t|}<M^{*} e^{\frac{i t}{\hbar} H_{1}} M e^{-\frac{i t}{\hbar} H}>d t
$$

Здесь $\gamma_{0}$ - естественная ширина возбужденного электронного уровня, $M$ - электронный матричный элемент; $H$ и $H_{1}$ - колебательные гамильтонианы соответственно в начальном и конечном электронных состояниях; $\langle\ldots\rangle \equiv \mathrm{Sp}\left(e^{-H / k T} \ldots\right) / \mathrm{Sp}\left(e^{-H / k T}\right)$.

C учетом ангармонизма третьего и четвертого порядка, используя операторы рождения $\left(a_{j}^{+}\right)$и уничтожения $\left(a_{i}\right)$ фононов, гамильтониан $H$ можно записать в виде суммы нулевого гамильтониана $H_{0}$ (который берется как гамильтониан невзаимодействующих осцилляторов) и возмущения $H^{\prime}$ :

$$
\begin{gathered}
H=H_{0}+H^{\prime} \\
H_{0}=\sum_{j} \hbar \omega_{i}\left(a_{j}^{+} a_{i}+\frac{1}{2}\right) ; \\
H^{\prime}=\frac{1}{6 i} \sum_{i j k} V_{i j k}\left(a_{i}-a_{i}^{+}\right)\left(a_{j}-a_{j}^{+}\right)\left(a_{k}-a_{k}^{+}\right)+ \\
+\frac{1}{24} \sum_{i j k s} W_{i i k s}\left(a_{i}-a_{i}^{+}\right)\left(a_{i}-a_{j}^{+}\right)\left(a_{k}-a_{k}^{+}\right)\left(a_{s}-a_{s}^{+}\right) .
\end{gathered}
$$

Здесь

$$
\begin{gathered}
V_{i j k}=\left(\frac{\hbar}{2}\right)^{3 / 2} \sum_{\alpha_{1} \alpha_{2} \alpha_{3}} v_{\alpha_{1} \alpha_{2} \alpha_{3}} \frac{e\left(\alpha_{1} \mid i\right) e\left(\alpha_{2} \mid j\right) e\left(\alpha_{3} \mid k\right)}{\sqrt{\omega_{i} \omega_{j} \omega_{k}}}, \\
W_{i j k s}=\left(\frac{\hbar}{2}\right)^{2} \sum_{\alpha_{1} \alpha_{2} \alpha_{0} \alpha_{4}} w_{\alpha_{1} \alpha_{2} \alpha_{3} \alpha_{4}} \frac{e\left(\alpha_{1} \mid i\right) e\left(\alpha_{2} \mid j\right) e\left(\alpha_{3} \mid k\right) e\left(\alpha_{4} \mid s\right)}{\sqrt{\omega_{i} \omega_{j} \omega_{k} \omega_{s}}},
\end{gathered}
$$

где $\boldsymbol{\alpha}$ нумерует компоненты декартовых координат ядер и сами ядра, $e(\alpha \mid j)$ - компонента собственного вектора динамической матрицы, относящаяся к осциллятору $j$ и декартовой координате $\alpha$, $v_{\alpha_{1} \alpha_{2} \alpha_{3}}$ и $w_{a_{1} \alpha_{2} \alpha_{2} \alpha_{4}}$ - коэффициенты в членах третьего и четвертого порядка в разложении адиабатического потенциала по степеням смещений атомов из положений равновесия.

Предполагаем, что электронный матричный элемент не зависит от колебательных координат ( $M=$ Const - приближение Кондона), а 
также, что при электронном переходе изменяются лишь положения равновесия ядер, а константы взаимодействия остаются прежними. В таком случае

$$
\begin{aligned}
H_{1} & =e^{\sum_{j} x_{0 j} \frac{\partial}{\partial x_{j}}} H e^{-\sum_{i} x_{0 j} \frac{\partial}{\partial x_{j}}}+\hbar \omega_{0}= \\
& =e^{\sum_{j} i z_{j}\left(a_{j}+a_{j}^{+}\right)} H e^{-\sum_{j} i z_{j}\left(a_{j}+a_{j}^{+}\right)}+\hbar \omega_{0},
\end{aligned}
$$

где $x_{i}$ - координата, $x_{0 j}-$ изменение положения равновесия осциллятора $j$ в электронном переходе; безразмерный параметр $z_{j}=x_{0 j} \sqrt{\omega_{j} / 2 \hbar}$ $\left(z^{2}{ }_{i}\right.$ - стоксовы потери, приходящиеся на осциллятор $j$, выраженные в числе его колебательных квантов); $\omega_{0}$ - частота чистоэлектронного перехода ( $\hbar \omega_{0}$ - энергетическое расстояние междуминимумами адиабатических потенциалов).

Веедем корреляционную функцию.

$$
I(t)=\left\langle e^{\sum_{j} i z_{j}\left(a_{j}+a_{j}^{+}\right)} e^{-\sum_{j} i z_{j}\left(a_{j}(t)+a_{j}^{+}(t)\right)}>.\right.
$$

Здесь $a_{j}^{+}(t)$ и $a_{j}(t)$ - операторы рождения и уничтожения фононов в гейзенберговском представлении: $a_{j}^{+}(t)=e^{\frac{i t}{\hbar}{ }^{+}} a_{j}^{+} e^{-\frac{i t}{\hbar} H}$, $a_{j}(t)=e^{\hbar^{i t} H} a_{j} e^{-\frac{i t}{\hbar} H}$

В таком случае

$$
I(\omega)=\frac{1}{2 \pi}|M|^{2} \int_{-\infty}^{\infty} e^{i t\left(\omega_{0}-\omega\right)-\gamma_{0}|t|} I(t) d t .
$$

В эффекте Мессбауэра в пренебрежении изменением параметров решетки при $\gamma$-переходе вероятность испускания $I_{y}(\omega)$, а также вероятность поглощения $\gamma$-кванта $I \varkappa(\omega)$ частоты $\omega$ (нормнрованные на еднницу) определяются следующим образом [7]:

$$
\begin{aligned}
& I_{u}(\omega)=\frac{1}{2 \pi} \int_{-\infty}^{\infty} e^{i\left(\omega-\omega_{0}\right) t-\gamma_{0}|t|} J(t) d t \\
& I_{\%}\left(\omega-\omega_{0}\right)=I_{y}\left(\omega_{0}-\omega\right) .
\end{aligned}
$$

Здесь $\hbar \omega_{0}-$ разность энергии уровней ядра, $\gamma_{0}-$ естественная ширина возбужденного ядерного уровня, а корреляционная функция $J(t)$ равна следующему:

$$
J(t)=\left\langle e^{\overrightarrow{i k} \vec{U}_{q(0)}} e^{-\overrightarrow{i k}} \vec{U}_{q(t)}\right\rangle,
$$


где $\vec{U}_{q}(t)$ - вектор тепловых смещений $\gamma$-радиоактивного ядра номера $q$ в гейзенберговском представлении, $\vec{k}-$ волновой вектор $\gamma$-кванта.

Выразив $U_{q}^{(v)}$ ( $v$ нумерует декартовы компоненты вектора) через операторы рождения и уничтожения фононов $a_{j}^{+}$и $a_{j}$,

$$
U_{q}^{(v)}=i \sum_{j} e(q, v / j)\left(\hbar / 2 m_{q} \omega_{j}\right)^{1 / 2}\left(a_{j}-a_{j}^{+}\right),
$$

получим $J(t)$ в виде

$$
J(t)=\left\langle e^{\sum_{j} z_{j}\left(a_{j}^{+}-a_{j}\right)} e^{-\sum_{j} z_{j}\left(a_{j}^{+}(t)-a_{j}(t)\right)}>,\right.
$$

где $z_{j}=\sum_{\nu} k_{\nu} e(q, v / j)\left(\hbar / 2 m_{q} \omega_{i}\right)^{1 / 2}$.

Таким образом, расчет электронноколебательных спектров примесных центров в кристаллах и расчет спектра $\gamma$-квантов в эффекте Мессбауэра сводятся соответственно к вычислению корреляционных функций $I(t)$ и $J(t)$, которые аналогичны.

В гармоническом приближении $I_{0}(t)=J_{0}(t)$, т. е. обе корреляционные функции в точности совпадают. Это следует из инвариантности колебательного гамильтониана $H_{0}$ относительно замены координат на импульсы (точнее в используемых нами обозначениях $H_{0}$ инвариантен относительно замены $i\left(a_{j}-a_{j}^{+}\right)$на $\left.\left(a_{j}+a_{j}^{+}\right)\right)$. В этом же нетрудно убедиться путем непосредственного расчета. Вычислим для этого в гармоническом приближении $\left(H=H_{0}\right)$ функцию $f_{j}(t)$

$$
f_{i}(t)=\left\langle e^{\alpha_{j}\left(a_{j}^{+}+\eta a_{j}\right)} e^{-a_{j}\left(a_{j}^{+}(t)+\eta a_{j}(t)\right)}>\right.
$$

где $\eta= \pm 1$

Используя тождество Вейля [8]

$$
e^{a a+\beta a^{+}}=e^{\frac{1}{2} \alpha \beta} e^{\beta a^{+}} e^{\alpha a}=e^{-\frac{1}{2} \alpha \beta} e^{\alpha a} e^{\beta a+},
$$

формулу (13) можно переписать в виде

$$
\begin{gathered}
f_{j}(t)=e^{\alpha_{j}^{2} \eta}<e^{\alpha_{j} a_{j}^{+}} e^{\eta \alpha_{j} a_{j}} e^{-\alpha_{j} a_{j}^{+}(t)} e^{-\eta \alpha_{j} a_{j}(t)}>= \\
=e^{\eta \alpha_{j}^{2} \mu_{j}}<e^{\alpha_{j} \mu_{j} a_{j}^{+}} e^{\alpha_{j} \mu_{j}^{*} a_{j}}>,
\end{gathered}
$$

если $a_{j}^{+}(t)=e^{i \omega_{j} t} a_{j}^{+}, \quad a_{j}(t)=e^{-i \omega_{j} t} a_{j}, \quad \mu_{j}=1-e^{i \omega_{j} t}$.

Разложив выражение, стоящее в $(10)^{\prime}$ под знаком $\langle\ldots\rangle$, в ряд, получим

7 ENSV TA Toimetised F-1 65. 


$$
f_{i}(t)=e^{\eta \alpha_{j}^{2} \mu_{j}} \overline{\sum_{r=0}^{n_{j}} \frac{\left(\alpha_{j}^{2} \eta\right)^{r}\left|\mu_{j}\right|^{2 r} n_{j} !}{\left(\eta_{j}-r\right) ! r ! r !}}=e^{\eta \alpha_{j}^{2}\left(\mu_{j}+\left|\mu_{j}\right|^{2} \vec{n}_{j}\right)}
$$

где черта сверху означает усреднение по колебательным состояниям, а $\bar{n}_{j}=\left(e^{\hbar \omega_{j} / k T}-1\right)^{-1}-$ среднее значение колебательного квантового числа $n_{i}$ при температуре $T$.

Подставим (13) в (7) и (10б), положив соответственно $\eta=1, \alpha_{j}=i z_{j}$ и $\eta=-1, \alpha_{j}=z_{j}$. Получим хорошо известные формулы Лэмба [9] для $J_{0}(t)$ и Лэкса [10] для $I_{0}(t)$, которые в используемых здесь обозначениях действительно совпадают и справедливы как для кристаллических, так и для локальных колебаний:

$$
I_{0}(t)=J_{0}(t)=\exp \left\{-\sum_{j} z_{j}^{2}\left[2 \vec{n}_{j}+1-\left(\vec{n}_{j}+1\right) e^{i \omega_{j} t}-\bar{n}_{j} e^{-i \omega_{j} t}\right]\right\} .
$$

Из этой формулы легко получаются характеристики бесфононной, а также других линий в рассматриваемых спектрах. Нам, в частности, понадобится формула для интенсивности линии, обязанной переходу с рождением $k_{\lambda_{1}}, k_{\lambda_{2}}, \ldots$ квантов локальных колебаний $\lambda_{1}, \lambda_{2}, \ldots$ ( $\lambda$ нумерует локальные, а $x$ - кристаллические колебания), но без изменения состояния кристаллических колебаний (см., например, $\left.\left[{ }^{11}\right]\right)$ :

$w_{k_{\lambda_{1}}, k_{\lambda_{2}}}, \cdots=\exp \left\{-\sum_{j} z_{j}^{2}\left(2 \bar{n}_{j}+1\right)\right\} \prod_{\lambda}\left(1+\frac{1}{\bar{n}_{\lambda}}\right) \frac{k_{\lambda}}{2} I_{k_{\lambda}}\left(2 z_{\lambda}^{2} \sqrt{\bar{n}_{\lambda}\left(\bar{n}_{\lambda}+1\right)}\right)$,

где $I_{k}(x)$ - функция Бесселя мнимого аргумента порядка $k$. Положив $k_{\lambda_{1}}, k_{\lambda_{2}}, \ldots=0, \pm 1, \pm 2, \ldots$, получим интенсивность бесфононной линии (все $k_{\lambda}=0$, см. [11]) и ее колебательных повторений, обуславливающих квазилинейчатую структуру соответствующих спектров (все эти линии в рассматриваемой модели колебаний, согласно выражениям (7), (10) и (14), имеют форму Лоренцовой кривой с радиационной шириной).

В случае отклонения от гармоничности колебаний $I(t) \neq J(t)$. Однако, как мы увидим ниже, если учесть влияние ангармонизма колебаний только на положение и ширину квазилиний, но пренебречь не столь сушественными поправками ангармонизма к вероятности переходов, то $I(t)=J(t)$. Тем самым будет показано, что имеется полное совпадение формул рассматриваемых явлений и с учетом ангармонизма колебаний.

\section{2. Слабая связь с колебаниями}

В ряде случаев взаимодействие электронного перехода в примесном центре с колебаниями решетки мало, т. е. малы стоксовы потери, выраженные в числе колебательных квантов, $z^{2}=\sum_{j} z_{j}^{2} \ll 1$. Так обстоит дело, например, во многих кристаллофосфорах с редкими землями в качестве активаторов, где электронные оболочки, между которыми совершается оптический переход, хорошо заэкранированы другими электронами. В эффекте Мессбауэра $z^{2}$ (здесь это энергия отдачи, выраженная в числе колебательных квантов) в некоторых случаях также 
существенно меньше единицы. Так, для $\gamma$-перехода в $\mathrm{Sn}^{119}$ с энергией $23,8 \kappa э в z^{2} \approx 0,03$, для $\mathrm{Fe}^{57 m}$ (с энергией перехода 14,4 кэв) и для $\mathrm{Tm}^{169 m}$ (с энергией перехода $8,4 \kappa э в$ ) $z^{2} \leq 0,01$. В таких случаях мы можем рассчитать $I(t)$ и $J(t)$ приближенно, разложив их в ряд по степеням $z_{i}$ и ограничившись несколькими первыми членами. Например, $I(t)$ с точностью до членов второго порядка по $z_{j}$ имеет вид

$$
I(t) \approx 1-\sum_{j} z_{j}^{2}<\left[\left(a_{i}+a_{j}^{+}\right)^{2}-\left(a_{i}+a_{j}^{+}\right)\left(a_{j}(t)+a_{j}^{+}(t)\right)\right]>.
$$

В данной работе мы не рассматриваем влияние ангармонизма колебаний на вероятности переходов. В таком случае

$$
I(t) \approx J(t) \approx 1-\sum_{j} z_{j}^{2}\left(2 \bar{n}_{i}+1\right)+\sum_{j} z_{j}^{2}\left[\left\langle a_{j}^{+} a_{j}(t)\right\rangle+\left\langle a_{j} a_{j}^{+}(t)\right\rangle\right] .
$$

Таким образом, задача определения $I(t)$ и $J(t)$ в этом случае сводится к расчету корреляционных функций $\varphi_{j}^{(1)}(t)=\left\langle a_{j}^{+} a_{j}(t)\right\rangle$ и $\varphi_{j}^{(2)}(t)=\left\langle a_{j} a_{j}^{+}(t)\right\rangle$.

Аналогичные корреляционные функции для идеальных кристаллов вычислены Кривоглазом [12] (см. также [13]) во втором приближении по ангармонизму третьего порядка с помощью метода двухвременных фунций Грина Боголюбова и Тябликова [14].

Ниже эти корреляционные функции находятся без ограничений на характер нормальных колебаний (последние могут быть и кристаллическими, и локальными). Используется также метод Боголюбова и Тябликова.

Обозначим как $\varphi_{j}^{(1)}(\omega)$ и $\varphi_{j}^{(2)}(\omega)$ Фурье-образы $\varphi_{j}^{(1)}(t)$ и $\varphi_{j}^{(2)}(t)$ соответственно. Согласно [ $\left.{ }^{14}\right]$,

$$
\begin{aligned}
& \varphi_{j}^{(1)}(\omega)=\underset{\varepsilon \rightarrow+0}{i}[(\$(\omega+i \varepsilon)-\Theta(\omega-i \varepsilon)], \\
& \varphi_{j}^{(2)}(-\omega)=e^{\hbar \omega / k T} \varphi_{j}^{(1)}(\omega),
\end{aligned}
$$

если $\mathbb{S}_{j}(\omega)$ есть Фурье-образ функции Грина $\mathbb{S}_{j}(\mathrm{t})$

$$
\begin{aligned}
\mathbb{B}_{i}(t)= & \ll a_{j} ; a_{j}^{+} \gg= \\
& =-i \Theta(t) \operatorname{Sp}\left\{a_{j}(t) a_{j}^{+}(0) e^{-H / k T}\right\} / \operatorname{Sp}\left(e^{-H / k T}\right)
\end{aligned}
$$

$\Theta(t)=1$, если $t>0 ; \Theta(t)=0$, если $t<0)$.

Поскольку здесь не рассматривается влияние ангармонизма на вероятности переходов, то статистическое усреднение можно проводить не с точной матрицей плотности $e^{-H / k T}$, а приближенно с более простой матрицей плотности $e^{-H_{0} / k T}$ :

$$
\begin{aligned}
\mathbb{S}_{j}(t) & \approx \mathbb{S}_{0 j}(t)=\ll a_{j} ; a_{j}^{+} \gg_{0}= \\
& =-i \Theta(t) \operatorname{Sp}\left\{a_{j}(t) a_{j}^{+}(0) e^{-H_{0} / k T}\right\} / \operatorname{Sp}\left(e^{-H_{0} / k T}\right)
\end{aligned}
$$


Пользуясь независимостью следа матрицы от представления, будем вычислять $\left(\$_{0 j}(t)\right.$ по состояниям невзаимодействующих осцилляторов.

Введем неусредненную статистически функцию Грина $\mathfrak{S}_{0 j}\left\{n_{i}\right\}(t)$ :

$$
B_{0 i\left\{n_{i}\right\}}(t)=-i \Theta(t)<\left\{n_{i}\right\}\left|a_{j}(t) a_{j}^{+}(0)\right|\left\{n_{i}\right\}>=\ll a_{j} ; a_{j}^{+} \gg_{\left\{n_{i}\right\}},
$$

где $\left\langle\left\{n_{i}\right\}|\ldots|\left\{n_{i}\right\}>\right.$ означает квантовомеханическое усреднение по состоянию невзаимодействующих осцилляторов с фиксированной совокупностью квантовых чисел их $\left\{n_{i}\right\}$. Усреднение $\mathscr{S}_{0 i} n_{i\}}(t)$ по колебательным состояниям дает $\mathscr{S}_{0 i}(t): \overline{\mathscr{S}_{0 i}\left\{n_{i}\right\}}(t)=\mathbb{S}_{0 i}(t)$.

Уравнение движения для функции Грина $\mathscr{S}_{0 j}\left\{n_{i}\right\}(t)$ в первом приближении по ангармонизму четвертого порядка имеет вид:

$$
\begin{aligned}
& i \frac{d \dot{S}_{0 j}\left\{n_{i}\right\}}{d t}=\delta(t) n_{j}+\omega_{j} \dot{S}_{0 j}\left\{n_{i}\right\}-\frac{1}{2 \hbar i} \sum_{s_{1} s_{2}} V_{j s_{1} s_{2}}\left[F_{j s_{1} s_{2}}^{(1)}-2 F_{j s_{1} s_{2}}^{(2)}+\right. \\
& \left.\quad+F_{j s_{1} s_{2}}^{(3)}\right]+\frac{1}{\hbar} \sum_{s} W_{j j s s}\left[n_{s}-\frac{1}{2}\left(n_{s} \delta_{s j}-1\right)\right] \mathcal{S}_{0 j\left\{n_{i}\right\}},
\end{aligned}
$$

где

$$
\begin{aligned}
& F_{j s_{1} s_{2}\left\{n_{i}\right\}}^{(1)}(t)=\ll a_{s_{1}}^{+} a_{s_{2}}^{+} ; a_{j}^{+} \gg\left\{n_{i}\right\} \\
& F_{j s_{1} s_{2}\left\{n_{i}\right\}}^{(2)}(t)=\ll a_{s_{1}}^{+} a_{s_{2}} ; a_{j}^{+} \gg\left\{n_{i}\right\} \\
& F_{j s_{1} s_{2}\left\{n_{i}\right\}}^{(3)}(t)=\ll a_{s_{1}} a_{s_{2}} ; a_{j}^{+} \gg\left\{n_{i}\right\} .
\end{aligned}
$$

Составим также уравнения движения для гринианов $F_{j s_{1} s_{2}\left\{n_{i}\right\}}^{(1)}, F_{j s_{1} s_{2}\left\{n_{i}\right\}}^{(2)}$ и $F_{j s_{1} s_{2}\left\{n_{i}\right\}}^{(3)}$, оставляя в них лишь члены, содержащие* $\mathscr{S}_{0 j}\left\{n_{i}\right\}$. Тем самым цепочка уравнений для неусредненных функций Грина обрывается. Отметим, что в случае усредненных по колебательным состояниям функций Грина процедура обрывания цепочки уравнений не может быть осуществлена корректно при наличии локальных колебаний ввиду конечности $V_{\lambda_{1} \lambda_{2} \lambda_{3}}$ и $W \lambda_{\lambda_{1} \lambda_{2} \lambda_{3} \lambda_{4}}$.

Для примера запишем уравнение для $F_{j s_{1} s_{2}\left\{n_{i}\right\}}^{(1)}(t)$ :

$$
\begin{aligned}
i \frac{d F_{j s_{1} s_{2}\left\{n_{i}\right\}}^{(1)}}{d t} & =-\left(\omega_{s_{1}}+\omega_{s_{2}}\right) F_{j s_{1} s_{2}\left\{n_{i}\right\}}^{(1)}-\frac{1}{\hbar_{i}} \operatorname{Si}_{0 j\left\{n_{i}\right\}} V_{j s_{1} s_{2}} \times \\
\times & {\left[1+n_{s_{1}}+n_{s_{2}}-\frac{1}{2} n_{j}\left(\delta s_{1 j}+\delta s_{2 j}\right)\right] . }
\end{aligned}
$$

* В уравнениях для функцин Грина $\mathscr{S}_{0 j\left\{n_{i}\right\}}(t)$ и гринианов $F_{j s_{1} s_{2}\left\{n_{i}\right\}}^{(1)}(t)$, $\boldsymbol{F}_{j s_{1} s_{2}\left\{n_{i}\right\}}^{(2)}(t)$ и $F_{j s_{1} s_{2}\left\{n_{i}\right\}}^{(3)}(t)$ отброшены члены, соответствующие двухфононным переходам, при которых рождаемый (поглощаемый) фонон вследствие ангармонического взаимодействия увлекает за собой в процесс еще один фонон. Такие переходы имеют порядок $z^{2} V^{2}$ либо $z^{2} W$ и могут быть более вероятнымн, чем двухфононные переходы порядка $z^{4}$, имеющие место уже в гармоническом приближении. Именно так обстоит дело, например, в случае ннфракрасного поглощения $U$-центрами в щелочногаломдных кристаллах $\left[{ }^{15}\right]$. 
Переходя к Фурье-представлению в полученных уравнениях, имеем после их решения

$$
\begin{aligned}
& \mathfrak{S}_{0 j\left\{n_{i}\right\}}(\omega)=\frac{1}{2 \pi} \frac{n_{j}}{\omega-\omega_{i}-R_{i\left\{n_{i}\right\}}(\omega)} ; \\
& R_{j\left\{n_{i}\right\}}(\omega)=R_{j\left\{n_{i}\right\}}^{(0)}(\omega)+R_{j\left\{n_{i}\right\}}^{(1)}(\omega) \\
& R_{j\left\{n_{i}\right\}}^{(0)}(\omega)=\frac{1}{2 \hbar^{2}} \sum_{s_{1} s_{2}} V_{j s_{1} s_{2}}^{2}\left(\frac{1+n_{s_{1}}+n_{s_{2}}}{\omega-\omega_{s_{1}}-\omega_{s_{2}}}+2 \frac{n_{s_{1}}-n_{s_{2}}}{\omega+\omega_{s_{1}}-\omega_{s 2}}-\right. \\
& \left.-\frac{1+n_{s 1}+n_{s_{2}}}{\omega+\omega_{s_{1}}+\omega_{s_{2}}}\right)+\frac{1}{\hbar} \sum_{s} W_{j j s s}\left(n_{s}+\frac{1}{2}\right) \\
& R_{j\left\{n_{i}\right\}}^{(1)}(\omega)=\frac{1}{2 \hbar^{2}} \sum_{s_{1} s_{2}} V_{i j i_{1}}^{\prime} V_{s_{1} s_{2} s_{2}}\left[\left(1+2 n_{s_{2}}\left(1-\delta_{s_{2} j}\right)\left(\frac{1}{\omega-\omega_{j}-\omega_{s_{1}}}-\right.\right.\right. \\
& \left.\left.-\frac{1}{\omega-\omega_{i}+\omega_{s_{1}}}\right)+n_{i} \delta_{s 2}\left(\frac{1}{\omega+\omega_{i}+\omega_{s_{1}}}-\frac{1}{\omega+\omega_{i}-\omega_{s_{1}}}\right)\right]-\frac{1}{2 \hbar} W_{i j i i} n_{j} .
\end{aligned}
$$

Для нахождения $\varphi_{j}^{(1)}(\omega)$ и $\varphi_{j}^{(2)}(\omega)$ необходимо еще усреднить $\mathfrak{S}_{0 ;}\left\{n_{i}\right\}(\omega)$ по колебательным состояниям осцилляторов. В случае функции Грина кристаллического осциллятора $\left(j=x_{i}\right)$ это усреднение сводится к простой замене всех $n_{i}$ на $\bar{n}_{i}$. Действительно, вклад любого отдельного осциллятора в $R_{j\left\{n_{i}\right\}}(\omega)$ здесь бесконечно мал при $N \rightarrow \infty(N-$ число ядер в кристалле). Это позволяет при последовательном усреднении сначала по одному осциллятору, затем по другому, третьему и т. д. каждый раз ограничиваться первым порядком по любому из слагаемых в (26б) и (26в). Последние же зависят линейно от каждого из $n_{i}$.

Усреднение по колебательным состояниям функции Грина, относящейся к локальному осциллятору $\left(j=\lambda_{i}\right)$, не так просто. Но и в этом случае результат усреднения по состояниям кристаллических осцилляторов сводится к замене всех $n_{\boldsymbol{\varkappa}_{i}}$ на $\bar{n}_{\boldsymbol{\varkappa}_{i}}$.

С учетом вышесказанного находим следующие формулы корреляционных функций $\varphi_{j}^{(1)}(\omega)$ и $\varphi_{j}^{(2)}(\omega)$ :

$$
\begin{gathered}
\varphi_{j}^{(1)}(\omega)=\operatorname{Av}_{\text {loc }} \frac{1}{\pi} \frac{\Gamma_{i}(\omega) n\left(\omega_{j}\right)}{\left(\omega-\omega_{j}-P_{i\left\{n_{\lambda}\right\}}(\omega)\right)^{2}+\Gamma_{j}^{2}(\omega)}, \\
\varphi_{j}^{(2)}(-\omega)=\frac{\bar{n}(\omega)+1}{\bar{n}(\omega)} \varphi_{j}^{(1)}(\omega),
\end{gathered}
$$

где $\mathrm{Av}_{\text {loc }}$ означает усреднение по стояниям локальных осцилляторов, $\Gamma_{j}(\omega)=\overline{\Gamma_{i\left\{n_{i}\right\}}(\omega)}$,

$$
\begin{gathered}
\Gamma_{i\left\{n_{i}\right\}}(\omega)=\frac{\pi}{2 \hbar^{2}} \sum_{s_{1} s_{2}} V_{j s_{1} s_{1}}^{2}\left[( 1 + n _ { s _ { 1 } } + n _ { s _ { 2 } } ) \left(\delta\left(\omega-\omega_{s_{1}}-\omega_{s_{2}}\right)-\right.\right. \\
\left.\left.-\delta\left(\omega+\omega_{s_{1}}+\omega_{s_{2}}\right)\right)+2\left(n_{s_{1}}-n_{s_{2}}\right) \delta\left(\omega+\omega_{s_{1}}-\omega_{s_{2}}\right)\right]
\end{gathered}
$$


а $P_{i\left\{n_{\lambda}\right\}}(\omega)$ - главное значение суммы (26а), определяющей $R_{i\left\{n_{i}\right\}}(\omega)$, если в последней все $n_{\varkappa_{i}}$ заменить на $\bar{n}_{\varkappa_{i}}$; кроме того, если $j-$ индекс кристаллического колебания, то следует и $n_{\lambda_{i}}$ заменить на $\bar{n}_{\lambda_{i}}$. В случае отсутствия локальных колебаний формулы (27) переходят в формулы, полученные в $\left.{ }^{13}\right]$.

$\Gamma_{i}(\omega)$ и $P_{j\left\{n_{\lambda}\right\}}(\omega)$ определяют затухание и сдвиг частоты осциллятора $j$, обусловленные ангармонизмом колебаний. Отметим, что для локальных колебаний сдвиг частоты изменяется на конечную величину при изменении квантового состояния какого-либо из локальных осцилляторов. Это обусловлено конечностыю параметров ангармонической связи между локальными колебаниями и их собственного ангармонизма.

Обычно по порядку величины $\Gamma_{j}(\omega)$ и $P_{j\left\{n_{\lambda}\right\}}(\omega)$ составляют - $(0,01-0,1) \omega_{j}$ (см. $\left.\left[{ }^{12,}{ }^{16}\right]\right)$, то есть существенно меньше $\omega_{j}$. Поэтому в первом приближении можно не учитывать зависимость их от $\omega$, положив $\Gamma_{j}(\omega) \approx \Gamma_{i}\left(\omega_{j}\right)=\Gamma_{j}$ и $\mathrm{P}_{i\left\{n_{\lambda}\right\}}(\omega) \approx P_{j}\left\{n_{\lambda}\right\}\left(\omega_{i}\right)=P_{j\left\{n_{\lambda}\right\}}$.

В таком случае искомые корреляционные функции $\varphi_{j}^{(1)}(t)$ и $\varphi_{j}^{(2)}(t)$ равны (формулы приводятся отдельно для кристаллических и локальных колебаний) :

$$
\begin{aligned}
& \varphi_{\varkappa_{i}}^{(1)}(t)=\bar{n}_{\varkappa_{i}} \exp \left\{-i \bar{\omega}_{\varkappa_{i}} t-\Gamma_{\varkappa_{i}}|t|\right\}, \\
& \varphi_{\varkappa_{i}}^{(2)}(t)=\left(\bar{n}_{\varkappa_{i}}+1\right) \exp \left\{i \bar{\omega}_{\varkappa_{i}} t-\Gamma_{\varkappa_{i}}|t|\right\}, \\
& \varphi_{\lambda_{i}}^{(1)}(t)=\mathrm{Av}_{\text {loc }} n_{\lambda_{i}} \exp \left\{-i \vec{\omega}_{\lambda_{i}}\left\{n_{\lambda}\right\} t-\Gamma_{\lambda_{i}}|\mathrm{t}|\right\}, \\
& \varphi_{\lambda_{i}}^{(2)}(t)=\operatorname{Av}_{\text {loc }}\left(n_{\lambda_{i}}+1\right) \exp \left\{i \vec{\omega}_{\lambda_{i}}\left\{n_{\lambda}\right\} t-\Gamma_{\lambda_{i}}|\mathrm{t}|\right\},
\end{aligned}
$$

где введены перенормированные частоты кристаллических и локальных осцилляторов: $\bar{\omega}_{\boldsymbol{x}_{i}}=\omega_{\varkappa_{i}}+P_{\boldsymbol{x}_{i}} ; \bar{\omega}_{\lambda_{i}}\left\{n_{\lambda}\right\}=\omega_{\lambda_{i}}+P_{\lambda_{i}\left\{n_{\lambda}\right\}}$.

С помощью полученных выражений (29) и (30) определяем по формуле (17) корреляционные функции $I(t)$ и $J(t)$. Подставив их в свою очередь в формулы (7) и (8), находим, что бесфононная линия в рассмотренной модели имеет те же характеристики, что и в гармоническом приближении. Кроме того, из (7) и (8) следует также, что $\varphi_{j}^{(1)}\left(\omega_{0}-\omega\right)$ и $\varphi_{j}^{(2)}\left(\omega-\omega_{0}\right)$ определяют интенсивность, положение, ширину и форму однофононных линий в спектре, если в последних $\mathrm{F}_{j}$ заменить на $\Gamma_{j}+\gamma_{0}$. Мы видим, что вследствие ангармонизма колебаний положение и ширина этих линий зависят от температуры (что, конечно, обязано температурной зависимости перенормированной частоты колебаний и константы затухания осцилляторов). В классическом пределе высоких температур эта зависимость линейна по $T$. При $T=0 \Gamma_{i}$ также отлично от нуля, что находится в соответствии с результатами работ $[12,13,16]$.

Вследствие того, что перенормированная частота $\bar{\omega}_{\lambda_{i}}\left\{n_{\lambda}\right\}$ локальных колебаний изменяется на конечную величину при изменении квантового состояния какого-либо из локальных осцилляторов, колебательные повторения бесфононной линии могут иметь внутренную структуру, если $\Gamma_{i}+\gamma_{0}$ существенно меньше изменения $\omega_{\lambda_{i}}\left\{n_{\lambda}\right\}$ при замене какого-либо 
из $n_{\lambda}$ на $n_{\lambda}+1 . *$ Это обстоятельство может привести и к некоторому дополнительному уширению рассматриваемых линий с температурой, если выполняется противоположное условие. Это уширение при высоких температурах также пропорционально $T$, при предельно низких температурах же оно исчезает (см. (27)).

Если имеется лишь одно локальное колебание, то однофононная линия, соответствующая рождению (поглощению) кванта этого колебания, состоит из равноотстоящих друг от друга компонентов, которые, как отмечалось, могут перекрываться. Расстояние между ними $\varepsilon_{\lambda}$ в энергетической шкале, согласно (27), равно

$$
\varepsilon_{\lambda}=\frac{3}{8} W_{\lambda \lambda \lambda \lambda}\left(\frac{\hbar}{\omega_{\lambda}}\right)^{2}-\frac{5}{24} \frac{V_{\lambda \lambda \lambda}^{2}}{\hbar \omega_{\lambda}}\left(\frac{\hbar}{\omega_{\lambda}}\right)^{3} .
$$

Положение наиболее интенсивной головной линии определяется формулой (26), если в последней положить $n_{\lambda}=0$ и $n_{x_{i}}$ заменить на $\bar{n}_{x_{i}}$. С удалением от нее интенсивность компонентов падает как $\exp \left\{-l \hbar \omega_{\lambda} / k T\right\} l$, где $l$ - номер компоненты.

Если пренебречь взаимодействием локального колебания с кристаллическими, то структуру рассматриваемых, однофононных линий легко найти и на основе решения задачи об уровнях энергии ангармониче. ского осциллятора [17].

\section{3. Обобщение на произвольную связь с колебаниями}

Откажемся теперь от ограничений на величину $z_{j}^{2}$. В целях упропения расчета не будем учитывать ангармонические операторы, дейстзующие одновременно больше, чем на один из локальных осцилляторов.

Рассмотрим эффект Мессбауэра. Считаем, что в кристалле имеется большое число $N^{\prime} \ll N \gamma$-радиоактивных ядер. Если имеются локальные колебания, то в этом случае их частоты можно считать $N^{\prime}$-кратно вырожденными. Введение нормальных координат этих колебаний не является однозначным. Поскольку мы пренебрегаем ангармоническими операторами, действующими одновременно больше, чем на одно локальное колебание, то вырождение сохраняется и с учетом ангармонизма колебаний и произвол в выборе нормальных координат локальных колебаний остается. Обычному введению их соответствует линейная комбинация декартовых смещений ядер около одной из дефектных областей в качестве одной из нормальных координат. Мы же выберем их таким образом, чтобы выполнялось условие $e_{\alpha}(p \mid j) \lesssim N^{\prime-\frac{1}{2}}$ для всех $p$ и $j$ (при этом каждая из нормальных координат локальных колебаний зависит от декартовых смещений ядер всех дефектных областей). В таком случае

$$
\begin{gathered}
J(t)=\prod_{i} \prod_{j}\left[1-z_{i} z_{i}\left\langle\left(a_{i}-a_{i}^{+}\right)\left(a_{i}-a_{j}^{+}\right)\right\rangle+\right. \\
\left.+z_{i} z_{j}<\left(a_{i}-a_{i}^{+}\right)\left(a_{j}(t)-a_{j}^{+}(t)\right)>\right]+\ldots,
\end{gathered}
$$

* Этот случай может легко реализоваться, когда имеется локальное колебание с частотой выше удвоенной предельной частоты фононного спектра. При этом ширина линий определяется ангармонизмом четвертого порядка во втором приближении, а расстояние между компонентами линии - ангармонизмом четвертого порядка в первом приближении и третьего порядка во втором приближении. 
или в пренебрежении влиянием ангармонизма колебаний на вероятно. сти переходов

$$
J(t)=\exp \left\{-\sum_{j} z_{j}^{2}\left[2 \vec{n}_{j}+1-<a_{j} a_{j}^{+}(t)>-<a_{j}^{+} a_{j}(t)>\right]\right\} .
$$

Формула (33) не зависит от выбора нормальных координат вырожденных колебаний, поскольку для них сумма $\sum_{j} e_{\alpha}^{2}(p \mid j)$ инвариантна по отношению к этому выбору ( $j$ здесь нумерует только вырожденные колебания) .

Корреляционные функции $\left\langle a_{i} a_{j}^{+}(t)\right\rangle$ и $\left\langle a_{j}^{+} a_{i}(t)\right\rangle$ в пренебрежении $V_{\lambda_{i} \lambda_{j} k}$ и $W_{i_{i} \lambda_{j} k s}$ согласно (29) (30) равны:

$$
\begin{aligned}
& \left\langle a_{i} a_{j}^{+}(t)\right\rangle=\left(\vec{n}_{j}+1\right) \exp \left\{i \overrightarrow{\omega_{j}} t-\Gamma_{j}|t|\right\}, \\
& \left\langle a_{j}^{+} a_{j}(t)\right\rangle=\bar{n}_{j} \exp \left\{-i \vec{\omega}_{j} t-\Gamma_{j}|t|\right\} .
\end{aligned}
$$

Подставим (34) в (33). В результате получаем

$J(t)=\exp \left\{-\sum_{j} z_{j}^{2}\left[2 \bar{n}_{j}+1-\left(\bar{n}_{j}+1\right) e^{i \bar{\omega}_{j} t-\Gamma_{j}|t|}-\bar{n}_{j} e^{-i \bar{\omega}_{j} t-\Gamma_{j}|t|}\right]\right\}$.

Проводя аналогичные преобразования $I(t)$, находим, что и в этом случае

$$
I(t)=J(t) .
$$

Рассмотрим на основе этих формул прежде всего влияние ангармонизма колебаний на бесфононную линию. Подставляя (35) и (36) в формулы (8) и (9), после интегрирования по $t$ видим, что в спектрах поглощения и излучения, как и в гармоническом приближении, имеются резонансные линии радиационной ширины. Однако интенсивность их определяется не формулой (15), в которой следует положить все $k_{\lambda}=0$, а формулой

$$
w_{0}=\exp \left\{-\sum_{j} z_{j}^{2}\left(2 \vec{n}_{j}+1\right)\right\}
$$

где $j$ нумерует как кристаллические, так-и локальные колебания. Тем самым функции Бесселя $\prod_{\lambda} I_{0}\left(2 z_{\lambda}^{2} \sqrt{\bar{n}_{\lambda}\left(\vec{n}_{\lambda}+1\right)}\right)$, обязанные локальным колебаниям, пропадают. Переходы. же, приводящие к появлению в формуле для $w_{0}$ в гармоническом приближении этих функций, имеют не радиационную, а обычно на несколько порядков бо́льшую ширину, определяемую константами затухания локальных осцилляторов $\Gamma_{\lambda}$. Следовательно, их надо исключить из бесфононной линии, хотя они могут давать достаточно узкую линию, которая может быть обнаружена на квазисплошном фоне переходов с участием кристаллических фононов.

Исчезновение функций Бесселя $\prod_{\lambda} I_{0}\left(2 z_{\lambda}^{2} \sqrt{\bar{n}_{\lambda}\left(\bar{n}_{\lambda}+1\right)}\right)$ в формуле для вероятности бесфононной линии можно понять следующим обра- 
зом. Из формулы (14) следует, что появление этих функций в формуле для $w_{0}$ обусловлено переходами с участием виртуальных фононов локальных колебаний (в переходе имеет место рождение и одновременное уничтожение некоторого числа локальных фононов; для кристаллических колебаний такие переходы не существенны, поскольку они не дают конечного вклада в вероятность $\left.w_{0}\right)$. Так как ангармонизм колебаний приводит к конечной ширине уровней локальных осцилляторов, то переходы с участием виртуальных фононов также должны иметь конечную ширину, определяемую константой затухания этих осцилляторов, и, следовательно, должны быть исключены из бесфононной линии.

Ангармонизм колебаний особенно существенно сказывается на колебательных повторениях бесфононной линии, соответствующих переходам с изменением квантовых чисел локальных осцилляторов.

Ширина этих квазилиний в отличие от гармонического приближения не является радиационной, а определяется их константой затухания $\Gamma_{\lambda}$. Например, при низких температурах $\left(\bar{n}_{\lambda} \ll 1\right)$ полуширина квазилинии $\left(\Delta E_{k_{\lambda}}\right)$, соответствующей рождению $k_{\lambda}$ квантов локального осциллятора $\lambda$, пропорциональна $\Gamma_{\lambda}$ и $k_{\lambda}: \Delta E_{k_{\lambda}}=\Gamma_{\lambda}\left|k_{\lambda}\right|$. При более высоких температурах возрастание полуширины с номером $\left|k_{\lambda}\right|$ квазилиний замедляется, но сами полуширины $\Delta E_{k \lambda}$ при этом увеличиваются. Отметим, что закон роста их с температурой является, вообще говоря, сложным и зависит от величины $z_{\lambda}^{2}-$ связи электронного и $\gamma$-перехода с соответствующими осцилляторами. Лишь при достаточно малом значении $z_{\lambda}^{2}$ этот закон в классическом пределе высоких температур является простым: $\Delta E_{k \lambda} \sim T$. Большему значению $z_{\lambda}^{2}$ соответствует более быстрый рост $\Delta E_{k \lambda}$ с температурой, чем линейный.

Положение квазилиний изменяется с ростом температуры благодаря зависимости $\bar{\omega}_{i}$ от $T$. При этом эквидистантность квазилиний в каждой колебательной последовательности сохраняется в рассмотренной нами модели. Этот результат связан с тем, что мы пренебрегли собственным ангармонизмом локальных колебаний, а также ангармонической связью их между собой. Учет последнего обстоятельства приведет к нарушению строгой эквидистантности и к возможности ангармонического расщепления квазилиний.

Приношу благодарность К. К. Ребане за руководство настоящей работой.

\section{ЛИТЕРАТ.У РА}

1. Т р и фонов Е. Д., ДАН СССР, 147, 826 (1963).

2. Ребане К. К., Хи жняков В. В., Опт. и спектр., 14, 362, 491 (1963); Р ебане К. К., Т ри фонов Е. Д., Хи жняков В. В., Тр. Ин-та физ, и астрон. АН ЭССР, № 27, 3, 1964.

3. Sils bee R. H., Phys. Rev., 128, 1726 (1962).

4. Кривоглаз М. А., Ж. эксперим. и теор. физ., 46, 637 (1964).

5. M a radudin A. A., Flinn P. A., Phys. Rev., 129, 2529 (1963).

6. Перлин Ю. Э., Усп. физ. наук, 80, 553 (1963).

7. S ingw i K. S., S jöla n der A., Phys. Rev., 120, 1093 (1960). (Перевод см. в сб́«Эффект Мессбауэра», ИЛ, М., 1962, стр. 193).

8. W e y 1 H., Z. f. Phys., 64, 1 (1929).

9. L a m b W. E., Phys. Rev., 55, 190 (1939). 
10. L a x M., J. Chem. Phys., 20, 1752 (1952). (Перевод см. в сб. «Проблемы физики полупроводников», ИЛ, М., 1957).

11. Реб ан е К. К., Хи жняков В. В., Тр. Ин-та физ. и астрон. АН ЭССР, № 20, 153 (1963).

12. Кри в огл а з М. А., Ж. эксперим. и теор. физ., 40, 567 (1961).

13. Ка щеев В. Н., Кривоглаз М. А., ФТТ, 3,1528 (1961).

14. Боголюбов Н. Н., Тябликов С. В., ДАН СССР, 126, 53 (1959); 3 уба ре в Д. Н., Усп. физ. наук, 71, 71 (1960).

15. Fritz B., Proc. Intern. Conf. Lattice Dynamics, Copenhagen, 1963.

16. Klemens P. G., Phys. Rev., 122, 443 (1961).

17. Л анд ау Л. Д., Л и фши ц Е. М., Квантовая механика, М., 1963, стр. 165.

\section{Институт физики и астрономии} Академии наук Эстонской ССР

\section{Поступила в редакцию} 9/V 1964

\section{HIZNJAKOV}

\section{KVASIJOONSETE ELEKTRONVONKUMISSPEKTRITE JA MÖSSBAUERI EFEKTI TEOORIA, VONKUMISTE ANHARMOONILISUST ARVESTADES}

Elektronvōnkumisüleminekuid lisanditsentriga kristallides vaadeldakse koos Mössbaueri efektiga. Näidatakse, et mõlema nähtuse analoogia säilib ka võnkumiste anharmoonilisuse arvestamisel, kui jätta kōrvale anharmoonilisuse parandus üleminekute tōenäosusse, mis toimuvad foononite tekkimisega (kadumisega). On leitud, et lokaalsete ostsillaatorite anharmooniline kustumine põhjustab foononivaba joone intensiivsust määrava valemi olulist muutumist. On saadud valemid, mis kirjeldavad anharmoonilisuse toimet lokaalsete foononüleminekutega joonte asendile ja laiusele. Vaadeldakse ühefonoonsete kvasijoonte anharmoonilist lōhenemist.

\section{HIZHNYAKOV}

\section{THEORY OF QUASILINEAR VIBRONIC SPECTRA AND THE MÖSSBAUER EFFECT WITH ANHARMONICITY}

The vibronic transitions in impure crystals are considered along with the Mössbauer effect. It is shown that the analogy of both effects will hold if the anharmonicity of vibrations is taken into account, but the anharmonicity effect in the transition probabilities is neglected. It is found that the anharmonical decay of local oscillators causes substantial change in the formula for the phononless line intensity. Formulas are obtained describing the eifect of anharmonicity upon the position and width of lines with creation and destruction of local phonons. The one-phonon quasilinear anharmonical splitting is considered. 